\title{
SD L-FILTER FOR FILTRATION OF IMAGES CORRUPTED BY MIXED NOISE IN FREQUENCY DOMAIN
}

Slower filter coefficients adaptation of adaptive LMS (Least Mean Square) filters in time domain can be improved by their constrained or unconstrained modifications, but not rapidly. Other alternative is its realisation in frequency domain, where filtered signal is de-correlated and adaptation algorithm converges faster. Based on idea of adaptation in frequency domain there was developed L-filter in frequency domain.

To the group of adaptive non-linear digital filters belong adaptive $L$-filters $(L-F)$, whose outputs are linear combination of order statistics. They have a simple design and they know how to adapt own coefficients to various noise types. For powerful suppression of mixed noise there was designed a signal-dependent filter structure, which consists of two L-Fs and one SID (Spatial Impulse Detector). The former suppresses noise in homogeneous regions, the latter preserves details of a filtered image.

This paper is devoted to the design of SD LMS L-F structure in frequency domain, which uses the SMD detector (Spatial Median Detector). The aim of this design is to obtain optimal parameters of SD LMS L-F such as threshold, level and adaptation step or optimal weight coefficients.

Keywords: adaptive LMS L-filters, Signal-Dependent structure, Spatial Impulse Detector, Fast Fourier Transform

\section{Introduction}

In real filtering of noisy data by various adaptive filters, the problem of faster coefficient adaptation must by solved. Rate of convergence depends on eigen values spread of autocorrelation matrix (ratio $\lambda_{M A X} / \lambda_{M I N}$ ) [1,5]. If this ratio is large, algorithm converges slowly. Faster convergence in time domain can be attained by parametric modifications of LMS algorithm (constrained or unconstrained), RLS algorithm, XLS algorithm etc. Other solution is based on de-correlation data principle in frequency domain.

For powerful suppression of mixed noise in still greyscale images there was developed a SD (Signal-Dependent) filter structure, which uses a version of L-filter (L-F) as single filter [1,3-5]. The output of L-F is defined as a linear combination of the order statistics in the input data observation [1,3-7]. Advantageous adaptation properties of filter design in frequency domain lead to design of SD L-F in this domain which is described in this paper.

The outline of this paper is as follows. Theory of LMS L-Filter in time and frequency domains are introduced in sections 2 and 3 , and in sections 4 and 5 SD LMS L-filters in both domains are described. Achieved results are described in section 6 and they are discussed in section 7 .

\section{L-filter, LMS L-filter in time domain}

Let a nonstationary observed signal $x$ in each pixel be defined by the original value $d$ and noise $n$

$$
x(m, n)=d(m, n)+n(m, n),
$$

where $m, n$ designate pixel coordinates in image.

A filtration window is defined by pixel centered around the central pixel $x(m, n)$ and they are ordered in a lexicographic ordering

$$
\begin{aligned}
& x_{i}=(x(m-\xi, n-\xi), \ldots, x(m, n), \\
& \ldots, x(m+\xi, n+\xi))^{T},
\end{aligned}
$$

where $\Xi=2 \xi+1$ defined a number of pixels in a row or column of square window

$$
x r_{i}=\left({ }_{1} x_{i},{ }_{2} x_{i}, \ldots,{ }_{N-1} x_{i},{ }_{N} x_{i}\right)^{T} .
$$

$x r_{i}$ is the vector of ordered pixels in ascending order. The pixel ${ }_{k} x_{i}$ represents $k$-th highest pixel of $i$-th input observation, where $k$ is from the range of 1 to $N\left(N=\Xi^{2}\right)$.

Output of L-filter (L-F) is for each as follows

$$
y_{i}=w^{T} x r_{i},
$$

where $w=\left(w_{1}, \ldots, w_{N}\right)^{1}$ is the coefficient vector of $\mathrm{L}-\mathrm{F}$ and in general, it can be obtained by Wiener-Hopf equation [1, 3-5]. In real applications, the computations of inversion of autocorrelation matrix $R_{i}^{-1}$ and correlation vector $p_{i}$ are more difficult, and this problem is usually solved by simple iterative algorithms. One from frequent algorithms is the LMS (Least Mean Square) algorithm, which is based on a SD (Steepest Descent) method. It minimises the MSE (Mean Square Error) parameter.

\footnotetext{
* Róbert Hudec

Department of Telecommunications, University of Žilina, Velký Diel, 01026 Žilina, Slovak Republic, tel.: +421 415132210 ,

E-mal: robert.hudec@fel.utc.sk
} 
Finally, the adaptation algorithm for adaptation of L-filter coefficient vector is given by

$$
\hat{w}_{i+1}=\hat{w}_{i}+2 \mu \varepsilon_{i} x r_{i} \text {. }
$$

\section{LMS L-filter in frequency domain}

Adaptation of highly corellated signals in time domain causes lower convergence of adaptation process. Its decorrelation in frequency domain makes the adaptation faster. There exist a lot of orthogonal transforms, which could be used. One of usually used transforms is fast version of DFT (Discrete Fast Fourier Transform) [2]. The scheme of adaptive L-filter in frequency domain $(\mathrm{LF} \div \mathrm{F})$ is shown in Fig. 1. It has some disadvantages as worse implementation, higher computation complexity (operation with complex numbers) [5]. Moreover, the adaptation of filter coefficient vector is the same as for L-F.

The output of LMS LF-F is given by

$y_{i}=\left|\operatorname{IDFFT}\left[w^{T} x r_{i}\right]\right|$.

\section{SD LMS L-filter in time domain}

An adaptive SD LMS L-filter consists of two single adaptive LMS L-filters and one SID (Spatial Impulse Detector) [4, 5]. Its structure is shown in Fig. 2.

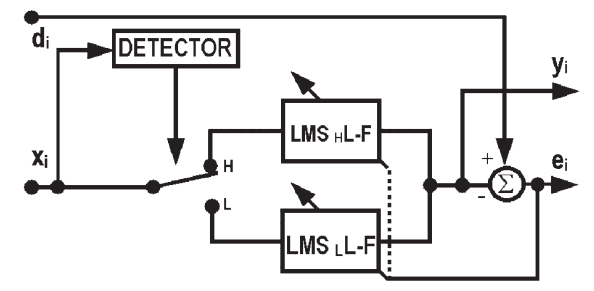

Fig. 2 The scheme of Signal-Dependent LMS L-filter in time domain

\subsection{SID (Spatial Impulse Detector)}

SID component serves as a switch between two LMS L-F. Moreover, if impulses are detected in the input sequence, these input data are processed by high-frequency segment. On the other hand, if in the input sequence any impulses or high-frequency components are not detected, the input data are processed by lowfrequency segment. The decision rule for SD L-filter in combination with spatial impulse detectors is given by

IF

$$
\sum_{k=1}^{N}{ }_{k} D_{i} \geq \text { Level }
$$

THEN $L M S_{H} L-F$

ELSE $L M S_{L} L-F$

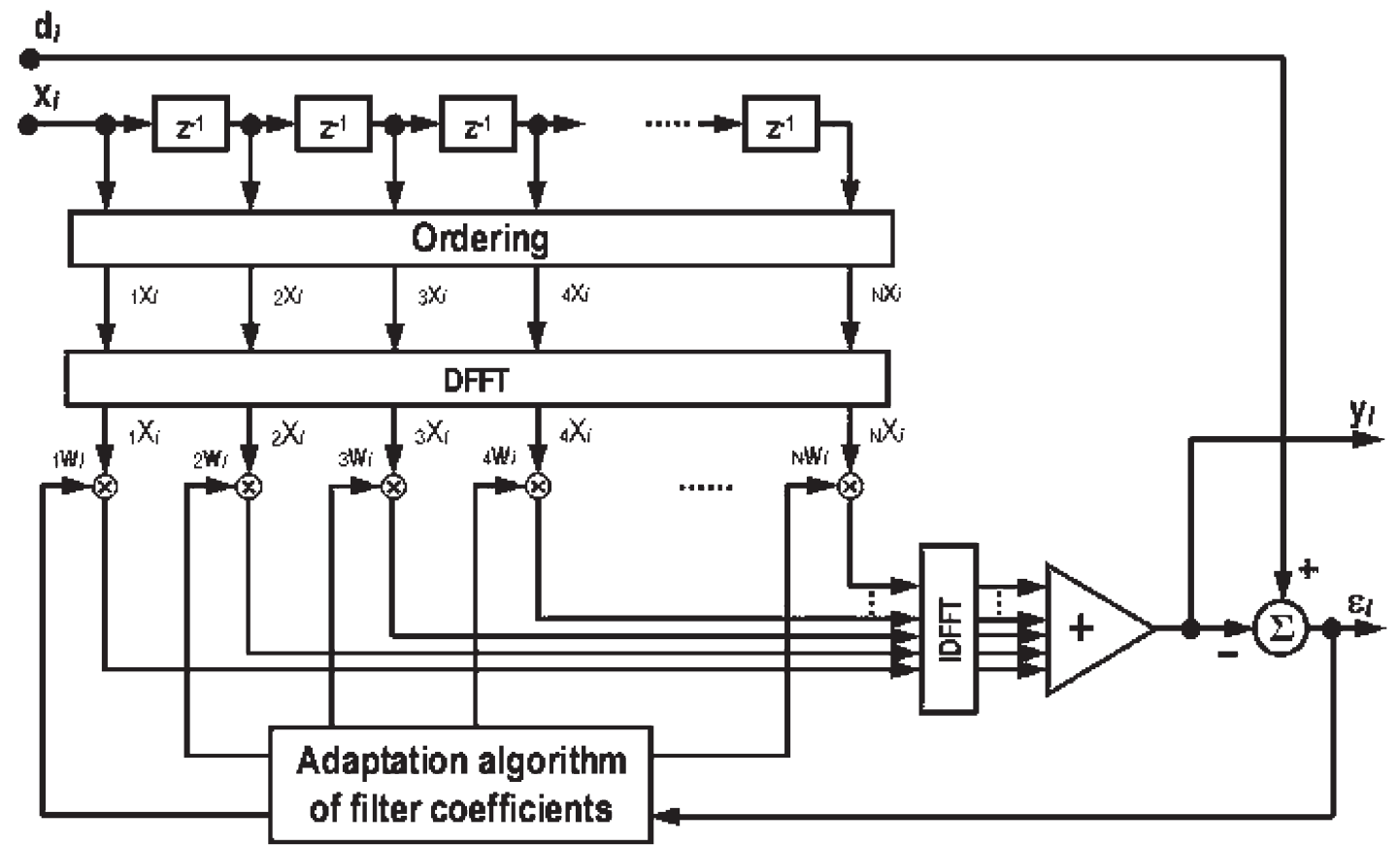

Fig. 1 The adaptive structure of LMS L-filter in frequency domain 
where ${ }_{k} D_{i}$ is the result of impulse detection for $k$-th image pixel in the $i$-th input vector. The value of level defines the number of detected impulses in the observed samples.

\subsubsection{SMD (Spatial Median Detector)}

The spatial median order detector (SMD) is one of the order statistic detectors family $[4,5]$. It is based on the following rule

IF

$$
\begin{gathered}
\mid \text { med }\left[x_{i}\right]-{ }_{k} x_{i} \mid \geq \text { Tolerance } \\
\operatorname{THEN}_{k} D_{i}=1 \\
\operatorname{ELSE}_{k} D_{i}=0
\end{gathered}
$$

If the difference in magnitude between $\mathrm{k}$-th and median input samples is more than the value of tolerance, this input sample is marked as a detected impulse. Its great advantage is a very simple design and smaller computation complexity.

\section{SD LMS L-filter in frequency domain}

SD LMS LF-F contains two LMS L-F, which work in a frequency domain (LMS LF-F). Moreover, the selected SID detects impulses in time domain. The filter scheme is shown in Fig. 3. One difference between filter realisation in time domain or frequency domain is that the adaptation equations (5) use in frequency domain transformed input data and in time domain non-transformed input data. Finally, the output of LF-F is realised by formula (6).

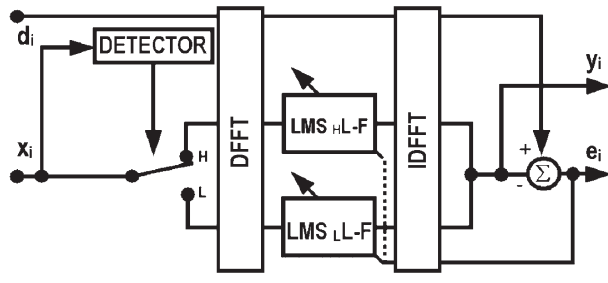

Fig. 3 The scheme of Signal-Dependent LMS L-filter in frequency domain

\section{Experimental results}

This section contains carried out experiments and achieved results. As the reference still image we use an image of Lena (Fig. $8 \mathrm{a})$. It was corrupted by mixed noise that consists of additive Gaussian white noise with standard deviation $\sigma=20$ and impulsive noise with probability $p=10 \%$ (Fig. $8 \mathrm{~b}$ ). The achieved filter results were evaluated mainly by the NR (Noise Reduction) parameter [3, 5]. The first experiment shows computational time dependencies from a filter window dimension and used L-filter.
From the filter mask dimension point of view for filtering of homogeneous areas, the computation complexity is important. As it is seen in Fig. 4, by increasing the filter window dimension, the adaptive versions have a lower computational complexity and they are suitable for realisations.

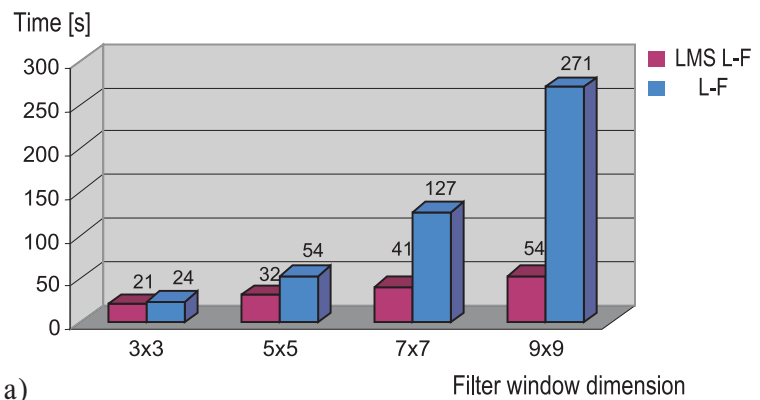

a)

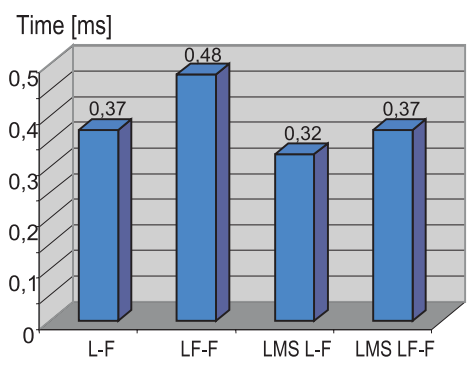

b)

Filter

Fig. 4 Computational time,

a) different square filter window of $L-F$ and $L M S L-F$, b) one iteration of $L-F, L F-F, L M S L-F$ and $L M S L F-F$

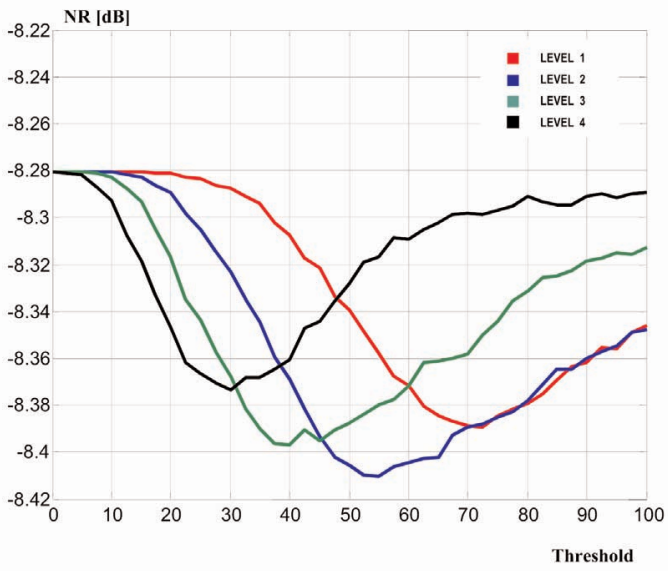

Fig. 5 NR dependencies of ${ }_{S M D} S D L-F$ for various levels

In Fig. 5 are shown NR dependencies from the threshold for various levels of ${ }_{\text {SMD }}$ SD L-F. The optimal set-up of SD L-F with SMD was achieved for the level $=2$ and threshold $=55$. 

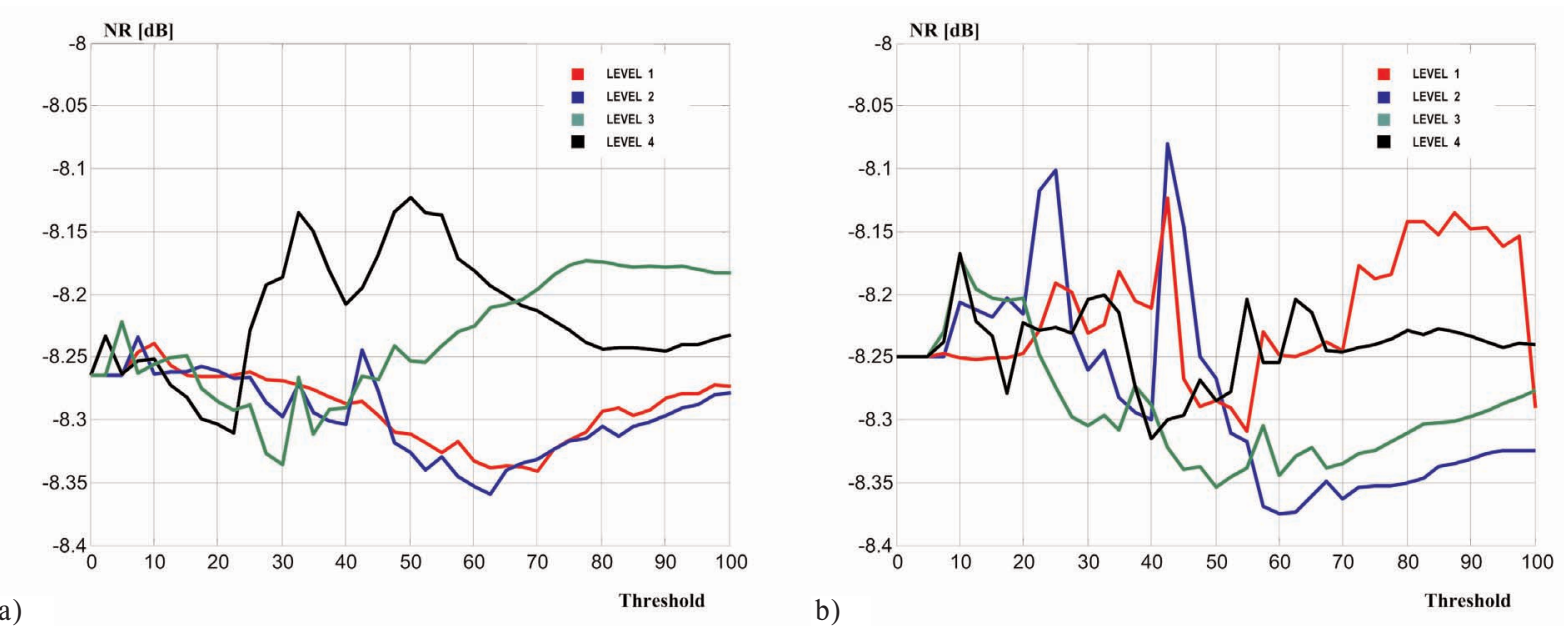

Fig. 6 NR dependencies of ${ }_{S M D} S D L M S L-F, \mu=1 \times 10^{-7}$,

a) time domain, b) frequency domain
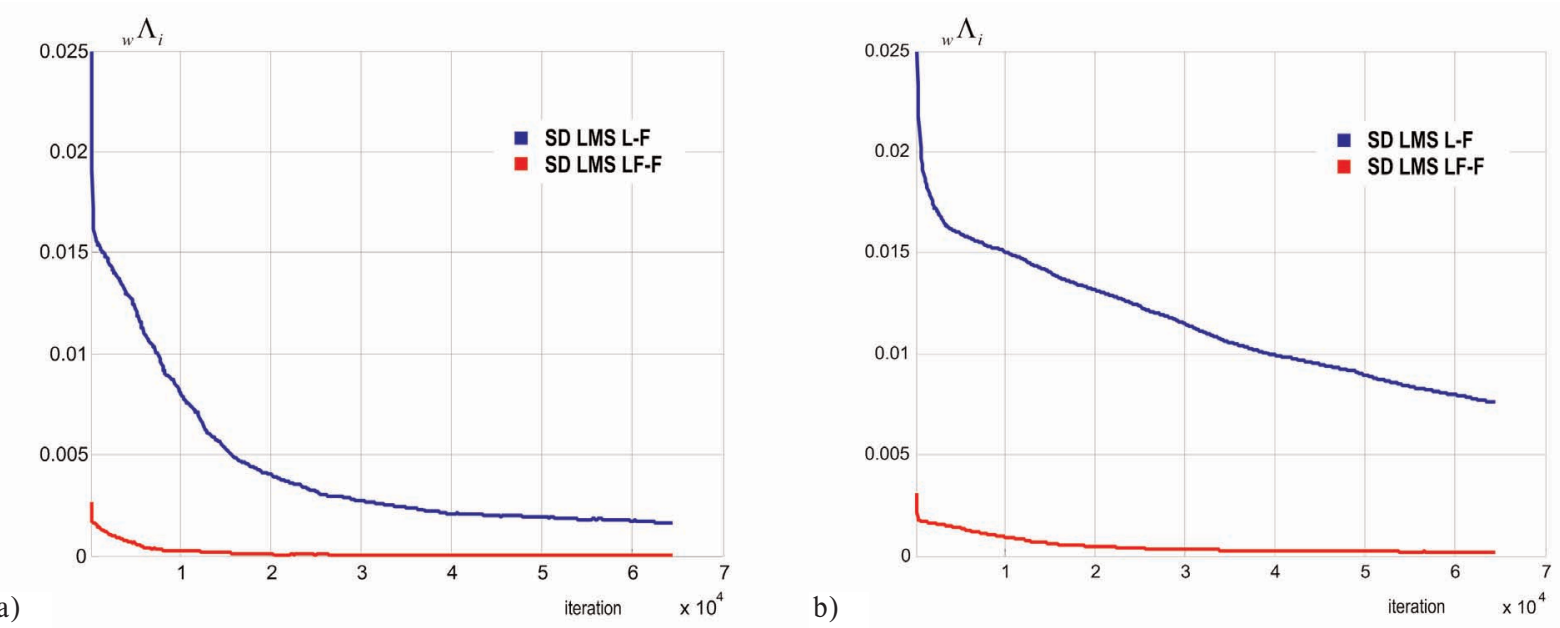

Fig. 7 Graphs of estimation error coefficient, a) $\left.\mu=1 \times 10^{-7}, b\right), \mu=1 \times 10^{-8}$

It is known that noise is more visible in homogeneous areas than in details. From this point, better noise filtering in homogeneous areas must be realised by a larger filter window. On the other hand, a better image structure preservation must be realised by a smaller filter window. A signal-dependent filter structure enables to realise filtrations of both aims separately. In practice, they have different filter windows and they depend on image properties. In experiments, equal 3 by 3 square filter windows were used. In Fig. 6 are shown NR dependencies from the threshold of SD LMS L-filter in both domains. It is seen that better filter results were achieved in a frequency domain where the SD filter converged faster. Both compared adaptive SD L-filters had for the level $=2$ displaced a threshold of about 60 .

The convergence measurement was realised by the coefficient of estimation error $[1,5]$

$$
{ }_{w} \Lambda_{i}=\frac{1}{N} \sum_{j=1}^{N}\left({ }_{j} \hat{w}_{i}-{ }_{j} w_{o}\right)^{2},
$$

where ${ }_{j} w_{o} j=1, \ldots, N$ consists of optimal filter coefficients for the used noise distribution and it is determined for one experiment realisation.

Convergence dependencies of SD filters in both domains for two adaptation steps are shown in Fig.7. It is evident that the version of SD L-F in frequence domain converges in first iteration under ${ }_{w} \Lambda_{i}<0.005$ for both adaptation steps. The same version in time domain converges under 0.005 level after 1500 iterations only for $\mu=1 \times 10^{-7}$. The limit of 0.005 interprets (at average) change of one coefficient from the optimal value about 0.3 .

Finally, in Fig. 8c, 8d the filtration results of LMS LF-F and SMDSD LMS LF-F are shown. Changes between single LF-F and ${ }_{\text {SMD }}$ SD LMS LF-F are not very visible, but after the set-up of optimal filter windows, the noise suppression effect will be evident. All experiments were realised by AMD K6-2/500MHz. 


\section{kOMNIKCCle}

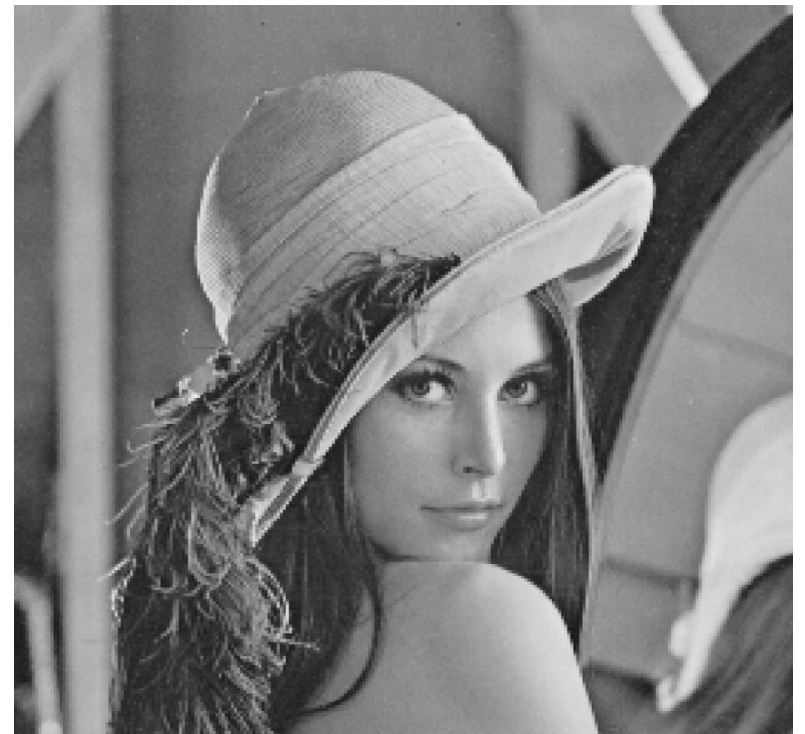

a)

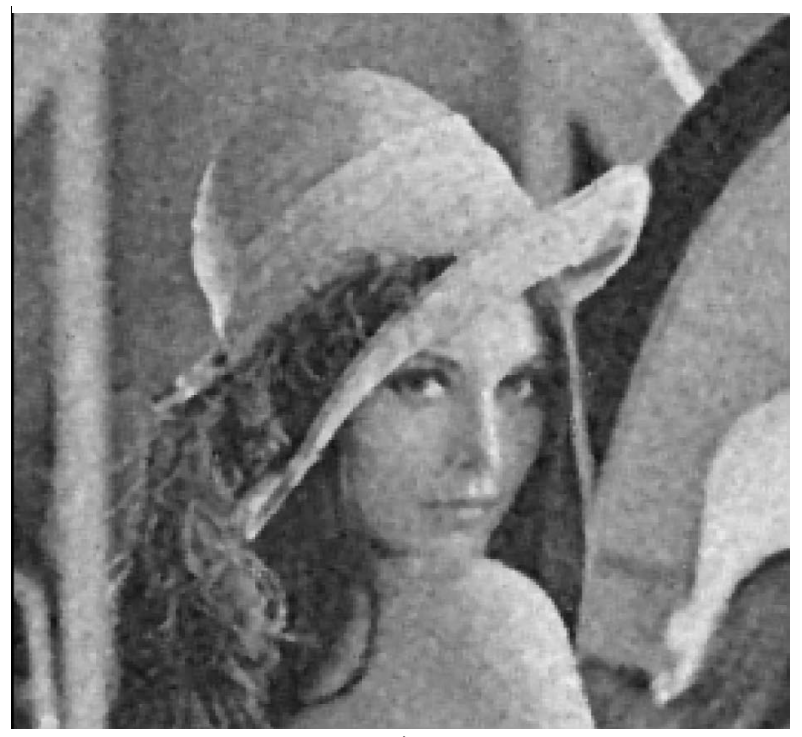

c)

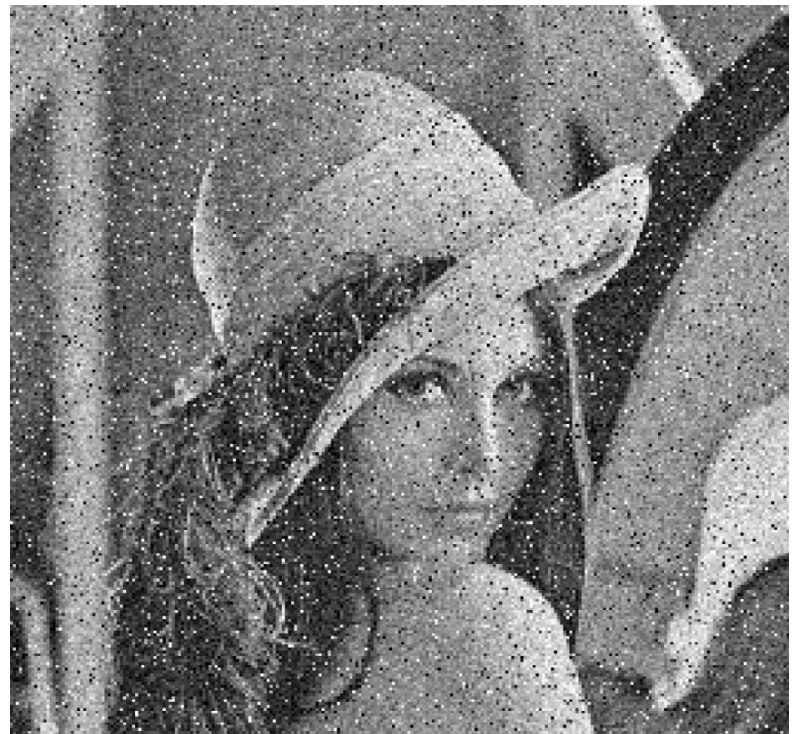

b)

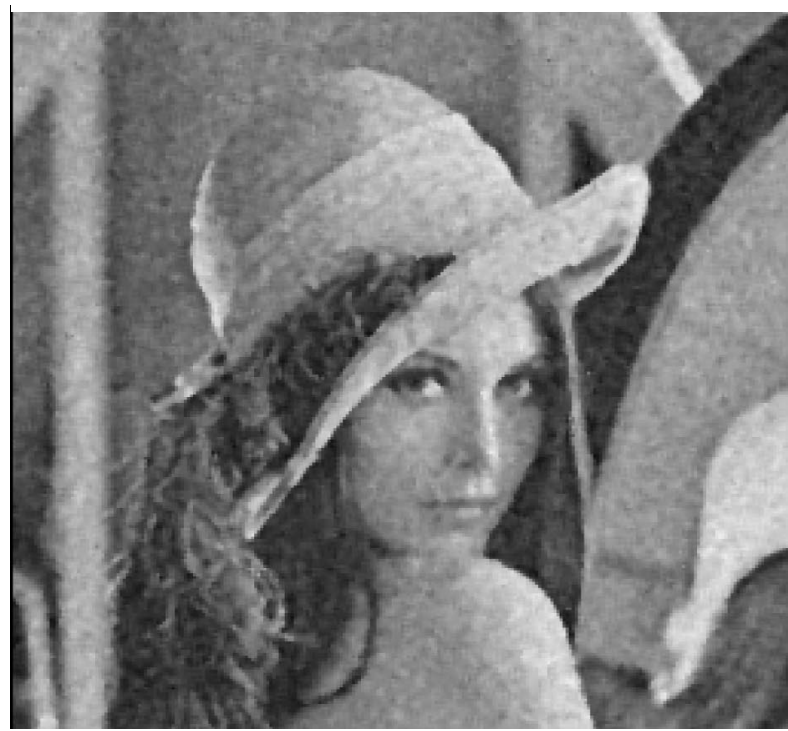

d)

Fig.8 Image Lena, a) original, b) noisy, c) filtered by LMS LF-F d) filtered by ${ }_{S M D} S D L M S L F-F$.

\section{Conclusion}

In this paper the signal-dependent L-filter in frequency domain was designed. The L-filter, as one of group of order statistic filters, which has suitable falicilities for various noise type suppression was used. Moreover, the signal-dependent filter structure multiplies its power. It could be assigned the fact, that for maximal noise suppression in homogeneous regions it uses a larger filter window and for edge preservation some pixels from input observation only.

Its adaptive realisation in time domain causes slower convergence of adaptation algorithm. The designed SD L-F in frequency domain employed a Spatial Median Detector in time domain, but adaptation of filter coefficient vector was realised in frequency domain. The proposed realization offered faster adaptation for the used adaptation steps.

Better filtration results could be obtained by determination of optimal detector window or by identification of optimal set-up filter windows.

\section{Acknowledgements}

This paper was supported by the state programme no. 2003 SP 51/028 09 00/028 0910 and VEGA-1/0140/03. 


\section{References}

[1] KOTROPOUlOS, C., PITAS, I.: Adaptive Nonlinear Filters for Digital Signal/Image Processing, Control and Dynamic Systems, C. T. Leondes, Vol. 67, San Diego CA, Academic press, pp. 263-317, 1994.

[2] BRIGHAM, E. O.: The Fast Fourier Transform and Its Applications, Prentice-Hall, Englewood Cliffs, NJ, 1988.

[3] KONTROPOULOS, C., PITAS, I.: Adaptive LMS L-Filters for Noise Suppression in Image, IEEE Transaction on Image Processing, vol.5, no. 12, pp. 1596-1609, Dec. 1996.

[4] HUDEC, R., MARCHEVSKÝ, S.: Extension of impulse detectors to spatial dimension and their utilisation as switch in the LMS L-SD filter, Radioengineering, vol. 10, no. 1, pp. 11-15, April 2001.

[5] HUDEC, R.: Filtration of noisy images corrupted by mixed noise by modifications of adaptive L-filters (in Slovak), PhD. Thesis, Košice, July 2002.

[6] MOUCHA, V., MARCHEVSKÝ, S., LUKÁČ, R., STUPÁK, CS.: Digital filtration of image signals. Vydavatel'stvo Vojenskej leteckej akadémie M.R. Štefánika v Košiciach. Košice, str. 365 (in Slovak), December 2000.

[7] GONZALEZ, R., WOODS, R.: Digital Image Processing, Addison-Wesley Publishing Company, 1992. 